\title{
Modified Approach to the Cervicofacial Rotation Flap in Head and Neck Reconstruction $^{\S}$
}

\author{
Warris A. Bokhari and Steven J. Wang* \\ Department of Otolaryngology - Head and Neck Surgery, University of California-San Francisco, San Francisco, CA, \\ USA
}

\begin{abstract}
Objectives: Large cutaneous defects of the cheek and external ear present a reconstructive challenge. While free tissue transfer has been increasingly used for such defects, many patients with these malignancies are poor candidates for lengthy reconstructive surgery. The cervicofacial and cervicothoracic advancement flaps have been previously described as a simple method for reconstructing defects of the face and neck. However, for large facial defects, these flaps typically require wide undermining with the final closure sometimes under tension. We describe our experience utilizing a modified approach to the cervicofacial rotation flap which has little tension of the defect closure and avoids incisions or undermining of tissue inferior to the clavicle.
\end{abstract}

Study Design: Retrospective chart review.

Methods: Sixteen patients underwent a modified cervicofacial rotation flap for immediate reconstruction of a cheek and/or total auriculectomy defect.

Results: Primary cutaneous malignancies were the most common surgical indication, followed by parotid tumors with overlying skin involvement. Five patients had total auriculectomy defects while 11 patients had defects involving cheek skin without auriculectomy. Defect diameter size varied from 5 to 12 centimeters. Two patients required skin grafting of the donor site. Four patients had minor wound dehiscences that were treated with local wound care and allowed to close by secondary intention.

Conclusions: We describe a modified approach to the cervicofacial rotation flap which minimizes undermining to provide a tension-free closure of the defect. Our approach is a simple, reliable method for coverage of large cutaneous defects involving the cheek and external ear.

Keywords: Head and neck cancer, reconstructive surgery, rotational flap.

\section{INTRODUCTION}

Large cutaneous defects of the cheek and external ear present a reconstructive challenge. While free tissue transfer has been increasingly used for such defects, many patients with these malignancies are poor candidates for lengthy reconstructive surgery. The use of a cervicofacial or cervicothoracic advancement flap in head and neck surgery was first described by Esser in 1918 and the technique has since evolved considerably [1]. Refinements have been primarily related to modified incision sites and a deeper plane of dissection.

In patients with malignant lesions involving the cheek skin and/or external ear, the cervicofacial flap can be used to reconstruct the cutaneous defect (Fig. 1A) [1,2]. The exposure required for elevation of this flap facilitates removal of the primary lesion, parotidectomy, and neck dissection. However, for large facial defects, this flap often

*Address correspondence to this author at the Department of Otolaryngology - Head and Neck Surgery, University of California-San Francisco, 2233 Post St., $3^{\text {rd }}$ floor, San Francisco, CA 94115, USA; Tel: (415) 885-7521; Fax: (415) 885-7546; E-mail.swang@ohns.ucsf.edu

${ }^{\S}$ Presented at the Meeting of the Western Section of the Triological Society, Inc, Rancho Mirage, CA, February 2, 2008. requires inferior extension of the incision over the chest to create a cervicothoracic flap (Fig. 1B). Elevation of a cervicothoracic flap typically involves wide undermining, with the final defect closure sometimes under tension. We describe our experience utilizing a modified approach to the cervicofacial rotation flap for large cheek defects in which there is little tension of the defect closure. We avoid dissection below the clavicle as with a standard cervicothoracic flap since we have rarely found infraclavicular dissection necessary to obtain adequate coverage of even large facial defects. We describe, in a series of large zone 1 and 2 cheek or external ear lesions, an anteriorly based cervicofacial flap with a modified incision pattern that provides suitable exposure for oncologic resection, sufficient tissue coverage of the defect, and results in a favorable aesthetic outcome.

\section{METHODS}

After obtaining institutional review board approval, we retrospectively reviewed 16 cases of anteriorly based cervicofacial rotation flaps performed for patients with cancer involving the cheek skin or external ear presenting at one of the University of California, San Francisco hospitals. All cases were patients of the senior author (SJW). These patients underwent immediate reconstruction for their cheek 
(A)

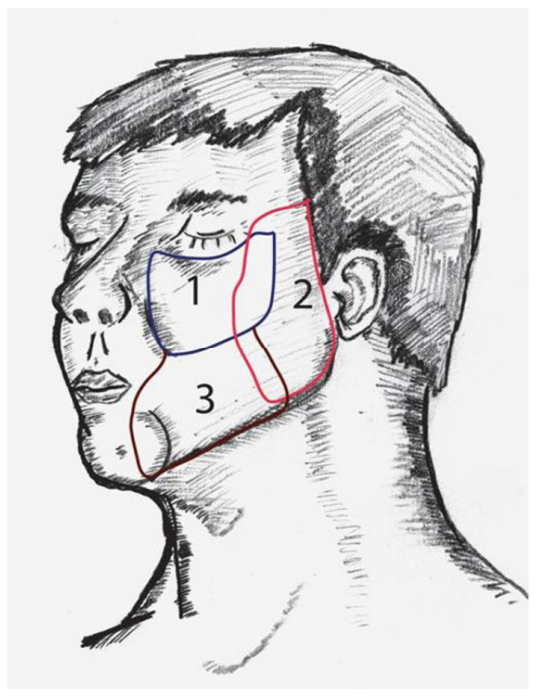

(B)
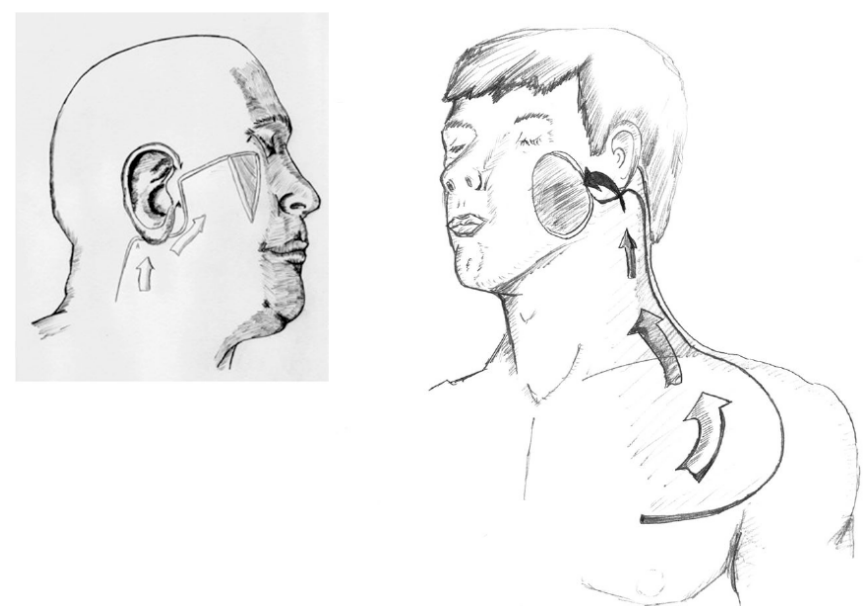

(C)

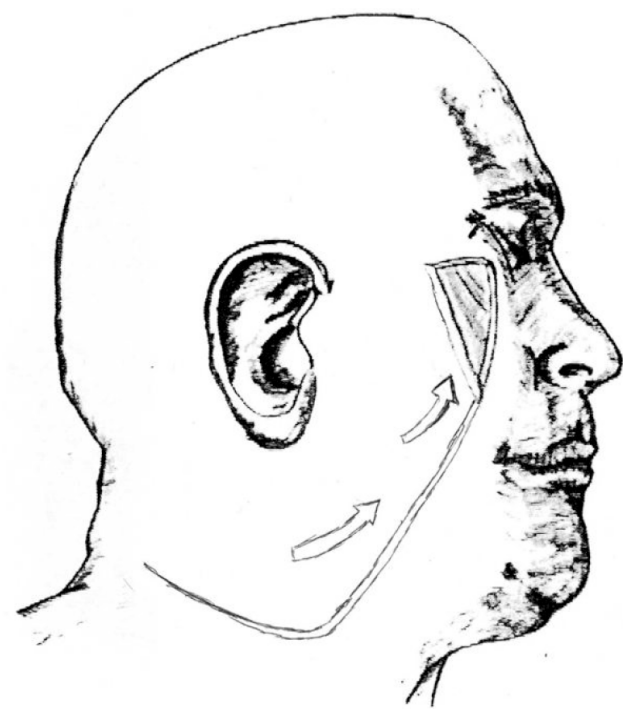

Fig. (1). Classification of cheek defects and commonly used incisions for flap repair. A) Zone $1-3$ for describing cheek defects; B) Common design for cervicofacial and cervicothoracic flaps; C) Illustration of the schematic design of a posteriorly based cheek flap reconstruction. and/or total auriculectomy defects. Patients who underwent microvascular free tissue transfer reconstruction, underwent no primary reconstruction, or were reconstructed solely with skin grafts were excluded from this study.

Medical charts were reviewed for patient demographic information, pathologic diagnosis, type of flap used, comorbid disease, and smoking history. The size of the defect was noted, and the location of the cheek defect was described according to the classification system of Cabrera and Zide (Fig. 1A) [3]. Length of operation and postoperative complications were also examined. When possible, photographs taken at the time of surgery and during postoperative visits were reviewed.

\section{Surgical Technique}

The flap is anteriorly based. For defects involving the medial cheek, the incision extends superolaterally in a slight upward direction above the zygomatic arch before continuing along the preauricular crease, in order to minimize the risk of ectropion of the lower eyelid (Fig. 2). For very large or lateral cheek defects, the incision extends from the lateral aspect of the resection margin inferior to the lobule and is carried across the mastoid tip into the hairline (Fig. 3). The width of the flap is approximately equal to the maximum diameter of the defect, so that there is no tension

(A)

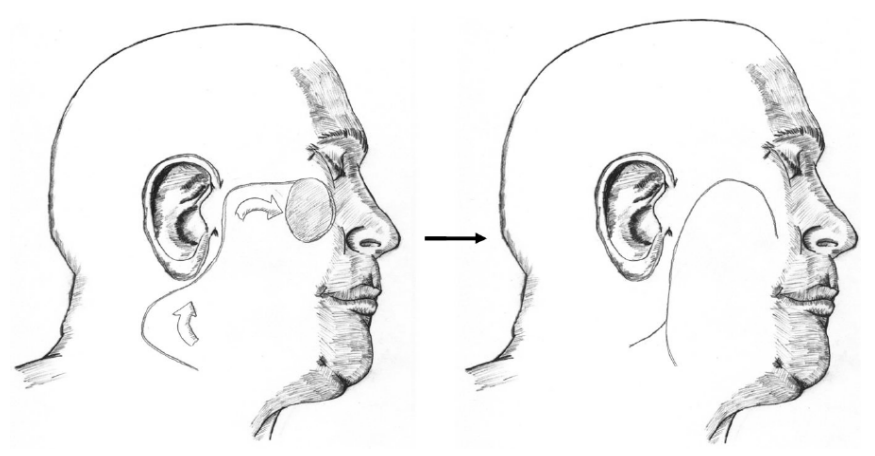

(B)

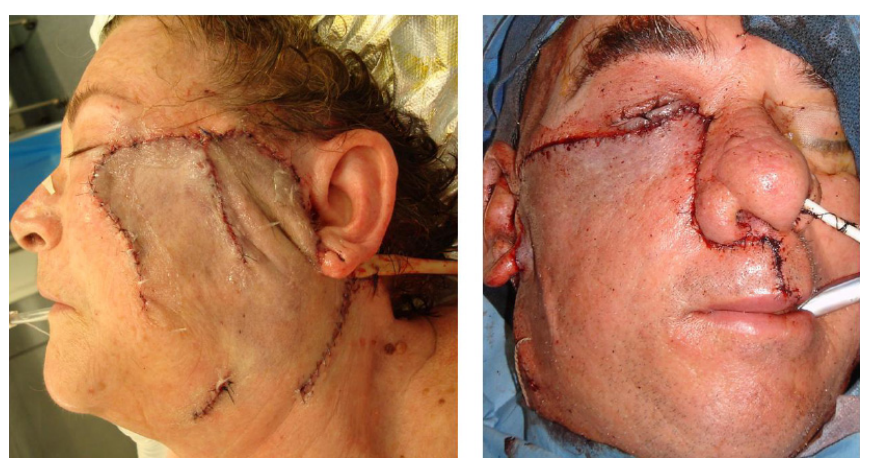

Fig. (2). Technique for repair of Zone 1 cheek defects. A) Schematic diagram; B) The patient on the left had closure of a medial cheek defect with a bilobed cervicofacial rotation flap. A sentinel lymph node biopsy of a level 1 node was simultaneously performed. The patient on the right had an extended radical maxillectomy with a $6 \mathrm{~cm}$ medial cheek skin defect. A cervicofacial rotation flap was combined with a Weber-Ferguson incision to accomplish tumor resection and cheek skin defect closure. 
(A)

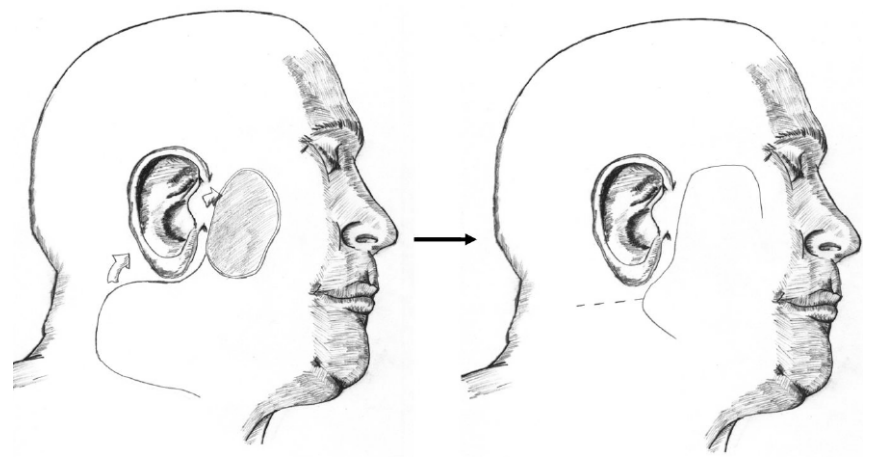

(B)

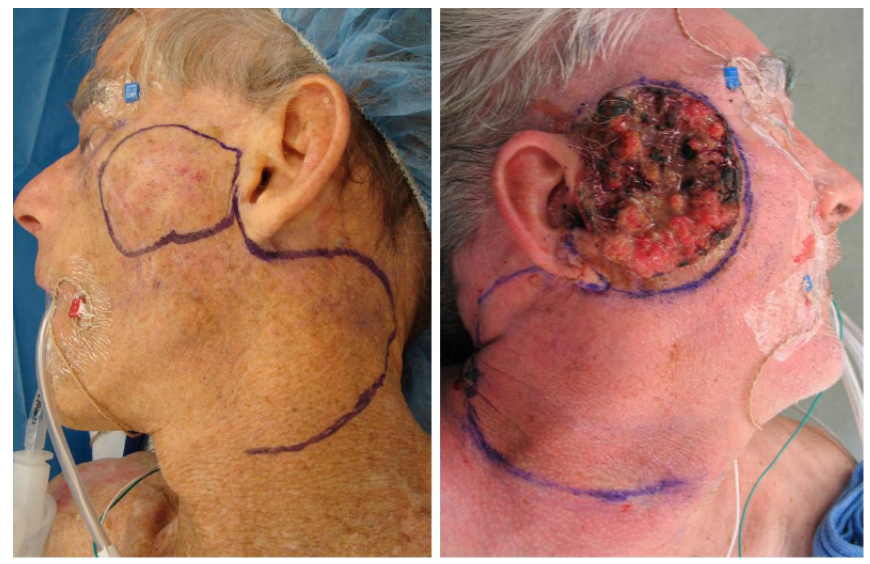

(C)
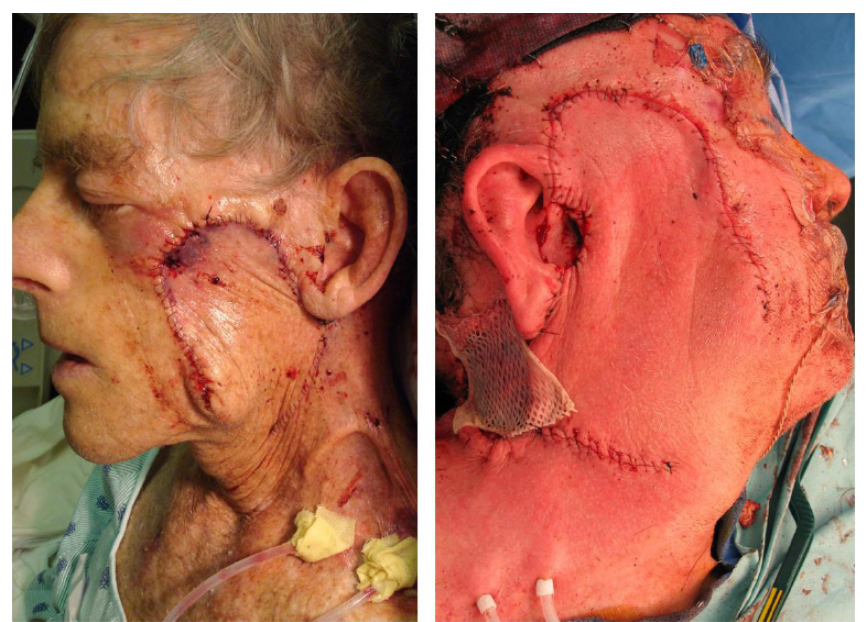

Fig. (3). Technique for repair of Zone 2 cheek defects. A) Schematic diagram; B) Flap design for two patients with large preauricular defect; C) Coverage of large pre-auricular defects with cervicofacial rotation flaps. For the patient on the left, the vertical dog-ear at the inferomedial aspect of the defect closure was left intact because of concern for the viability of the tip of the flap. However, no post-operative wound breakdown occurred. For the patient on the right, a skin graft was utilized for flap donor site coverage.

of the flap over the defect. Taking the tip of the flap beyond the hairline also facilitates a tension free closure with the drawback being the transfer of hair-bearing skin to the cheek, which may necessitate depilation later. The inferior incision is then carried medially at approximately ninety degrees for a distance as necessary to offer sufficient rotation for the flap to reach the defect. A similar flap design is used for the total auriculectomy defect except that the flap is taken more inferiorly and posteriorly in the neck (Fig. 4). The donor area can generally be closed primarily, often with a $\mathrm{V}$ $\mathrm{Y}$ advancement, after wide undermining down to but not inferior to the clavicle. On occasion, a skin graft for the donor site may be used. A vertical dog-ear in the inferomedial aspect of the defect closure is typically formed (Fig. 3C), which may be excised at the time of reconstruction, or later if there is concern for compromise of the flap. For the flap elevation, dissection is performed in a plane deep to the superficial musculoaponeurotic system (SMAS) in the face and deep to the platysma muscle in the neck [4].

(A)

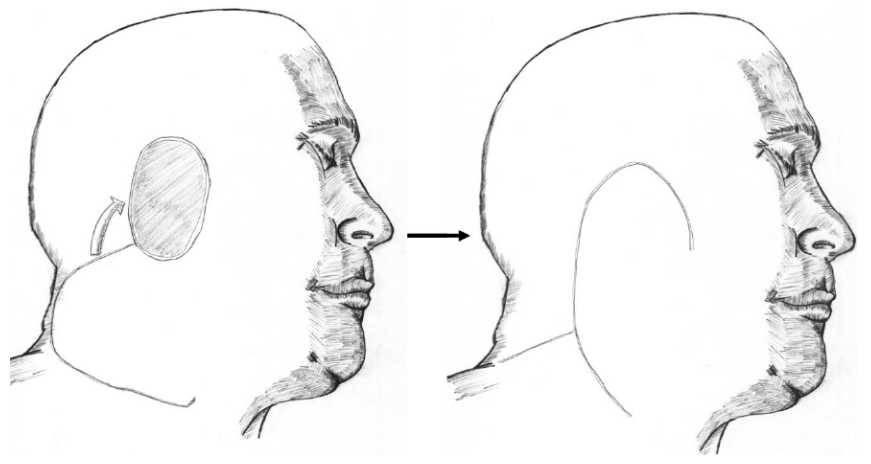

(B)

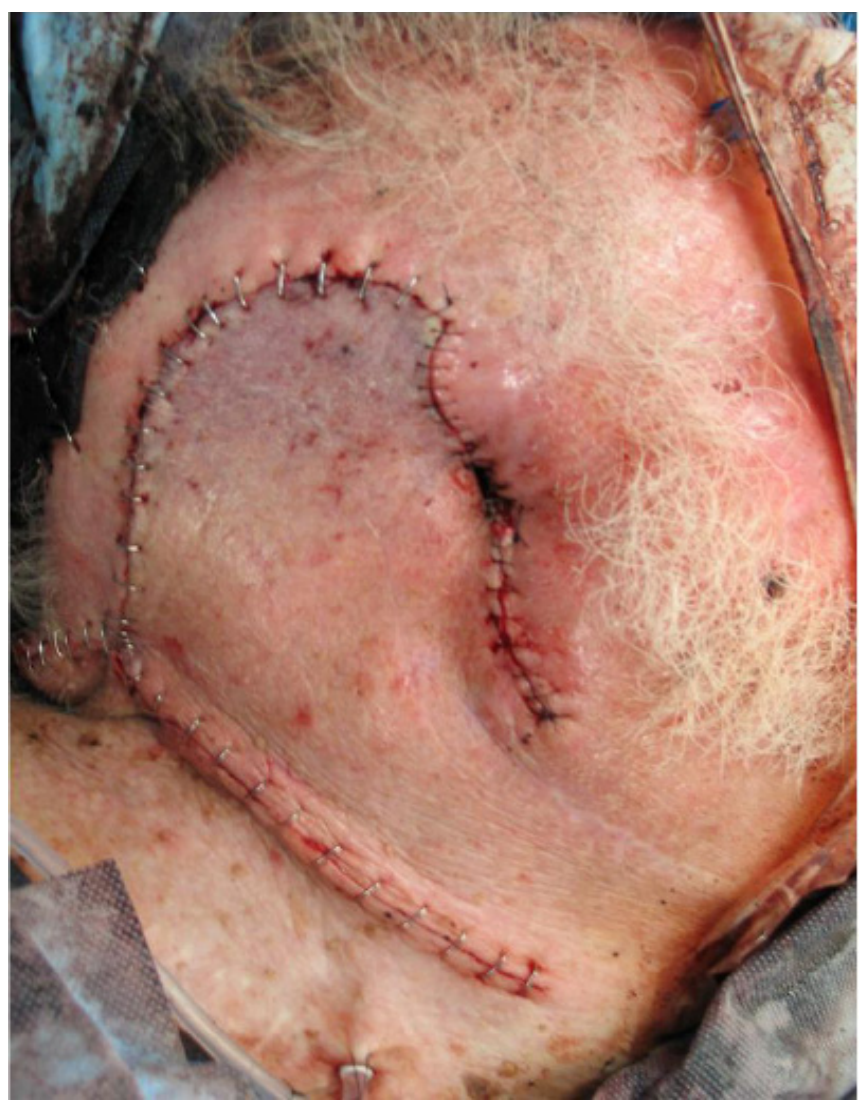

Fig. (4). Technique for repair of Zone 2 cheek plus total auriculectomy defects. A) Schematic diagram; B) Coverage of total auriculectomy defect with a cervical rotation flap. 
Table 1. Patients in Our Series

\begin{tabular}{|c|c|c|c|c|c|c|c|}
\hline Patient & Sex & Age & Pathology & Defect & Diameter & Other Procedures Done & $\begin{array}{l}\text { Wound Dehiscence } \\
\text { (Time to Healing) }\end{array}$ \\
\hline 1 & M & 70 & MCC skin & Zone 2 & $4 \mathrm{~cm}$ & Superficial parotidectomy & \\
\hline 2 & M & 80 & SCC skin & External ear & $8 \mathrm{~cm}$ & Superficial parotidectomy, ND & \\
\hline 3 & M & 60 & SCC skin & Zone 2 & $12 \mathrm{~cm}$ & Superficial parotidectomy, ND & Donor site ( 6 weeks) \\
\hline 6 & M & 70 & SCC ear & External ear & $8 \mathrm{~cm}$ & Superficial parotidectomy, ND, temporal bone resection & Flap (2 months) \\
\hline 7 & M & 65 & Melanoma & External ear & $8 \mathrm{~cm}$ & None & \\
\hline 8 & $\mathrm{M}$ & 50 & SCC maxilla & Zone 1 & $6 \mathrm{~cm}$ & Superficial parotidectomy, ND, maxillectomy, orbital exenteration & \\
\hline 9 & $\mathrm{M}$ & 65 & SCC parotid & Zone 2 & $6 \mathrm{~cm}$ & Superficial parotidectomy, ND & Donor site (3 weeks) \\
\hline 12 & $\mathrm{~F}$ & 75 & Melanoma & Zone 2 & $5 \mathrm{~cm}$ & Superficial parotidectomy, SLN biopsy & \\
\hline 13 & $\mathrm{~F}$ & 67 & Melanoma & Zone 1 & $5 \mathrm{~cm}$ & SLN biopsy & \\
\hline 14 & $\mathrm{~F}$ & 67 & Melanoma & Zone 1 & $5 \mathrm{~cm}$ & SLN biopsy & Flap (3 weeks) \\
\hline 15 & M & 70 & SCC parotid & Zone 2 & $6 \mathrm{~cm}$ & Radical parotidectomy & \\
\hline 16 & $\mathrm{M}$ & 70 & SCC skin & External ear & $8 \mathrm{~cm}$ & Superficial parotidectomy, ND & \\
\hline
\end{tabular}

\section{RESULTS}

The 16 patients in our series are described in Table $\mathbf{1 .}$ Thirteen patients underwent surgery for primary cutaneous malignancies of the cheek or ear. Five patients had squamous cell carcinoma of the skin, 4 patients had melanoma, 2 patients had basal cell carcinoma, and 2 patients had Merkel cell carcinoma. Two patients had parotid malignancies with extension to involve the overlying pre-auricular skin. One patient had a primary squamous cell carcinoma of the maxilla with extension to involve the medial cheek skin. There were 13 men and 3 women, with a mean age of 70 (range 50-80).

Six patients had defects involving Zone 1 (medial cheek), 5 patients had defects involving Zone 2 (pre-auricular cheek) without involvement of the ear, and 5 patients had total auriculectomy defects. Defect diameter ranged from 5 to 12 $\mathrm{cm}($ mean $-7 \mathrm{~cm})$. All the flaps were entirely elevated above the clavicle. The donor sites were closed primarily except in two cases where a split-thickness skin graft was utilized (Fig. 3C). Four patients $(25 \%)$ had minor wound dehiscences. In two patients, there was partial loss at the tip of the flap, and two patients had wound breakdown in the donor site area (Fig. 5C). The 4 cases of wound dehiscence were treated with local wound care and allowed to close by secondary intention. All eventually closed satisfactorily without requirement for additional surgery or hospitalization. Typical healing time for wound dehiscences was from 3 to 6 weeks with the longest being 2 months. There did not appear to be a correlation between wound breakdown and increasing size of the defect. The one patient in our series who had a lateral temporal bone resection and a total auriculectomy suffered a wound dehiscence (Fig. 5G). When the cheek defect also includes the lateral skull base, such as after temporal bone resection, we found the cervicofacial rotation flap to be inadequate. In such cases, we now favor a musculocutaneous pedicled flap or free flap. There were no instances of unintentional facial nerve paralysis or spinal accessory nerve injury related to flap elevation.

Regarding length of operation, because most patients also underwent other simultaneous procedures such as parotidectomy, neck dissection or sentinel lymph node biopsy, it is difficult to retrospectively determine the contribution of the cervicofacial rotation flap to the overall length of the operation. However, certainly the exposure provided by flap elevation facilitated performing these other procedures. Thus in most cases, it is likely that the cervicofacial rotation flap added minimal time to the overall length of surgery.

The cervicofacial rotation flap provided reliable coverage of the cheek and/or total auriculectomy defect in all cases. The long-term functional and aesthetic outcomes for these flaps were typically excellent (Fig. 5). All patients were satisfied with the results of the reconstruction. One patient with a zone 1 lesion was bothered by hair growth at the tip of the flap and underwent subsequent laser depilation treatment.

\section{DISCUSSION}

Cabrera and Zide described an approximate system for describing the location of a lesion of the cheek (Fig. 1A) [5]. Zone 1 refers to suborbital defects. These lesions fall within an area bounded by the inferior orbital margin superiorly, the nasolabial crease medially and the gingival sulcus inferiorly. 
(A)

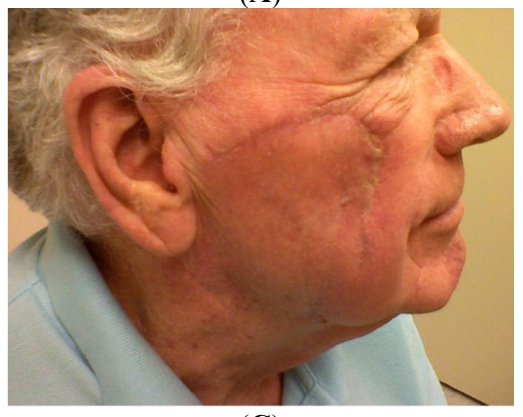

(C)

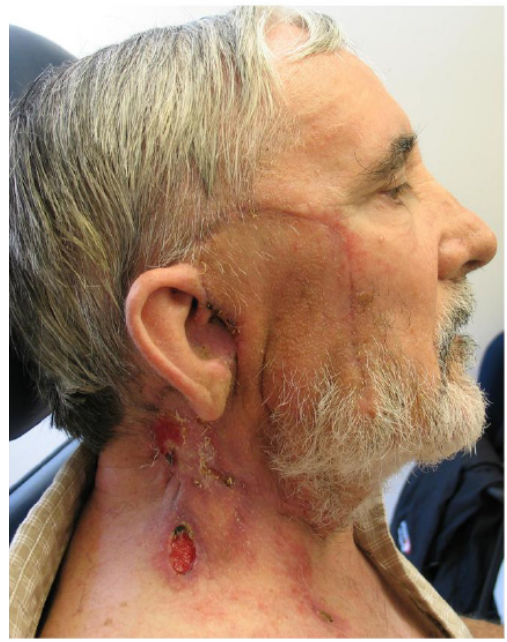

(E)

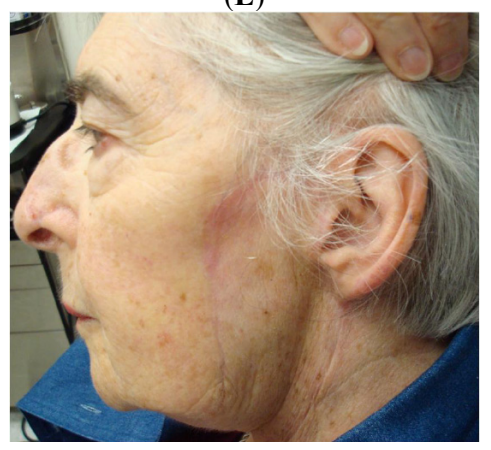

(B)

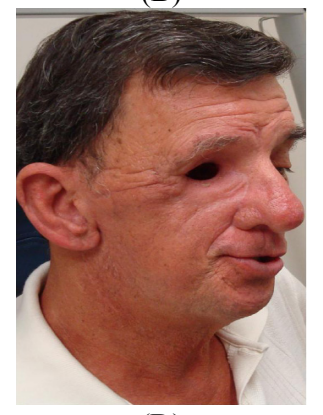

(D)

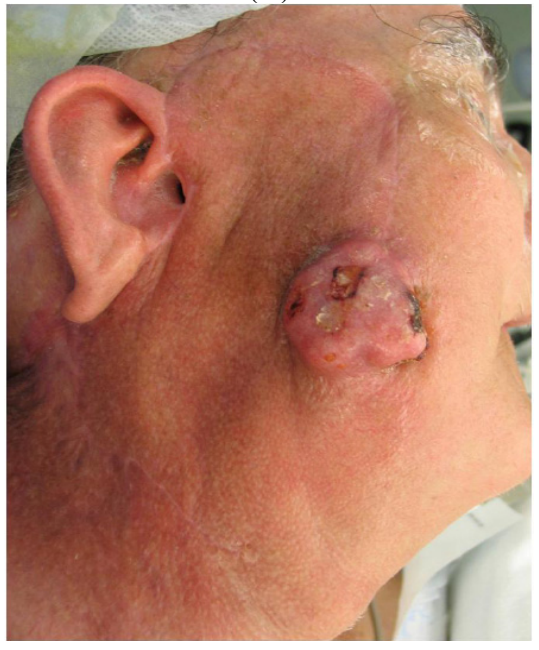

(F)

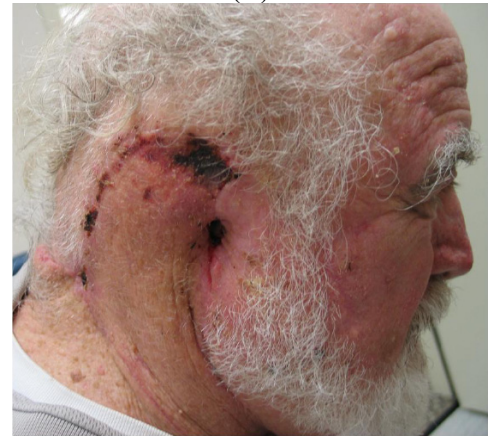

(G)

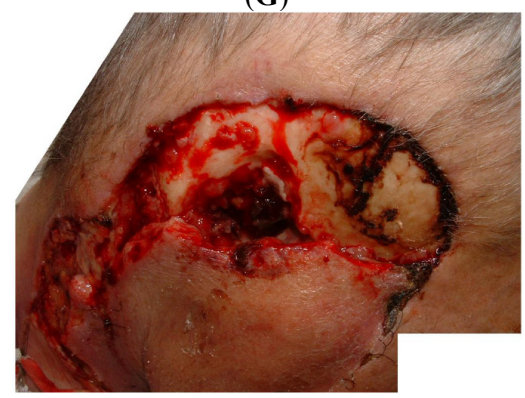

Fig. (5). Post-operative outcomes of cervicofacial rotation flaps. A) Zone 1 flap reconstruction at 2 months; B) Zone 1 flap reconstruction at 9 months of the second patient shown in Fig. (2B); C) Zone 2 flap reconstruction at 1 month of the second patient shown in Fig. (3B, 3C). A minor wound dehiscence of the donor site is closing by secondary intention; D) Zone 2 flap reconstruction at 5 months of the second patient shown in Fig. (3B, 3C). The patient had an anterior-inferior margin recurrence despite clear surgical margins and post-operative radiation therapy. The donor site wound dehiscence has closed; E) Zone 2 flap reconstruction at 6 months; F) Total auriculectomy defect flap reconstruction at 2 weeks. Although there is some duskiness of the tip of the flap, no wound dehiscence occurred; G) Distal skin loss of cervicofacial flap in patient undergoing reconstruction of a total auriculectomy and lateral temporal bone defect. This complication was treated with local wound care, and complete healing by secondary intention was successfully achieved 2 months later. We no longer advocate cervicofacial flaps for the reconstruction of cheek defects that also involve the lateral skull base. 
The lateral border extends up the medial border of the sideburn and joins the lateral canthus along an imaginary line. Zone 2 refers to preauricular defects. A lesion falling in zone 2 is bounded by the lateral border of the sideburn descending inferiorly to the angle of the mandible following it medially, then curving superomedially over the malar prominence, and finally extending superolaterally toward the lateral canthus. Zone 2 has overlap with zones 1 and 3 . Zone 3 refers to buccomandibular defects and defines an area in the lower cheek region. It is bordered laterally by the midpoint of the mandible, the lower lip medially, gingival sulcus superiorly, and extends out to its lateral border that overlaps with zone 2 .

There are many possible approaches to reconstruction of a cheek defect. The principles of reconstructing a cheek unit differ from reconstruction of a central facial unit where it is considered preferable to reconstruct the entire unit and excise surrounding tissue to avoid distracting asymmetry [6]. Replacement of the cheek as a whole aesthetic unit may preserve symmetry but is not particularly practical as it may result in the removal of large areas of potentially viable tissue [7]. Reconstruction of cheek lesions will vary according to the size and location of the defect. Small lesions less than $3 \mathrm{~cm}$ in diameter may be excised and closed primarily. Lesions larger than this dimension if closed primarily would cause distortion of the surrounding facial features impairing cosmesis and perhaps function [8]. Cheek defects from 3 to $5 \mathrm{~cm}$ in diameter may be closed satisfactorily with local flaps from within the cheek unit. For cheek defects larger than $5 \mathrm{~cm}$, however, one must generally consider using regional flaps [9]. Regional flaps offer excellent color match and are less visually prominent than skin grafting where the resultant skin texture may be uneven $[6,10]$. One stage reconstructions are generally favoured over delayed flaps as they impart less short-term morbidity for the patient.

Designing flaps for facial defects involve considering several key factors, including the location and size of the lesion, the surrounding structures and their function, the contour of the aesthetic unit, the preservation of symmetry, and whether concomitant neck dissection and/or parotidectomy are to be performed. The shape of the resulting defect will affect what repair is attempted. It is often convenient to excise additional tissue to allow for ease of closure, for instance circular defects may be converted to ellipses that are easier to close primarily. The orientation of the defect will dictate how the flap is applied; low lying horizontal defects are better suited to cervical advancement flaps and vertical ones by rotation advancement [6].

Various anteriorly based superficial subcutaneous flaps have been described for all 3 zones of the cheek with good aesthetic results [11-13]. For zone 1 lesions, the incision line for the flap extends to the posterolateral hairline. The infraorbital lesion is excised and the large mobile flap tissue is rotated and advanced to fill the defect. In men, this may cause alteration of the beard line. Cook et al. presented a series of 14 repairs of all 3 zones using a bilobed subcutaneous anteriorly based cervicofacial flap [11]. The incision lines ran infraorbitally, descending on the anterior aspect of the sideburn to run in the pretragal sulcus. The second lobe was post-auricular. The flap was bilobed to maximize the available tissue. Skin covering defects at the lower eyelid had to be thinned to provide a good texture match. The results were largely reported as excellent with complications of ectropion and poor wound healing in two cases. The authors noted that smoking status of the patients tended to impair healing.

Kaplan and Goldwyn described a posteriorly based cervicofacial advancement rotation flap supplied by the maxillary arteries and its branches [10]. The scar lines were camouflaged in the nasolabial groove. Ten cases were reported between 1967 and 1976, and good outcomes were achieved. This flap does, however, place scars toward the midline (Fig. 1C). Additionally, the vascular supply to the flap is not better than that of an anteriorly based flap.

Review of the literature finds several common strategies that have evolved over the years to improve the viability and reduce the risk of complications of the cervicofacial flap. To prevent ectropion, Crow and Crow proposed that any flap in the region below the eye should have its superior margin sutured at a point falling within a triangle bounded by the eyebrow, the lateral canthus, and the superior attachment of the ear to the face. ${ }^{14}$ Any flap with a superior margin below this triangle is likely to cause ectropion based on scar contraction and the weight of the flap. This description of an upcurving incision to prevent ectropion is found repeatedly throughout the literature $[14,15]$.

To minimize the risk of flap necrosis, several groups advocate a deep plane dissection of the flap. Specifically, elevation of the flap in a plane that is deep to the SMAS layer in the face and deep to the platysma muscle in the neck is done to improve the flap's vascularity. Elevating in this deeper plane is the basis of the commonly performed deepplane rhytidectomy [16]. Anteriorly based flaps in the deep plane draw their blood supply from branches of the submental and facial arteries. Moore et al. note that this plane is more avascular and permits a greater degree of tension to be employed in closure [17]. These authors described 13 cheek defect repairs using anteriorly based deep-plane rotational flaps in a 33 patient retrospective study. Fifteen of these patients received a cervicothoracic flap owing to a large facial defect. The authors note that distal extension into the thoracic region did not adversely affect patient outcomes or increase severity of complications. Tan and Mackinnon reported a similar series of 18 patients with defects that were repaired by an anteriorly based deep plane cervicofacial rotation flap [18]. Division of the facial suspensory ligaments was described as key to achieving greater flap mobility. The donor defects were all closed primarily, by z-plasty or V-Y advancement.

In designing a cervicofacial flap, dissecting deep to the platysma is said to enhance the vascularity of the flap by including the perforators from the underlying facial artery, improving flap survival and decreasing risk of distal tip necrosis. It has been suggested that deep-plane composite flaps are particularly useful for high-risk patients who smoke or have diabetes mellitus [2,18]. Facial nerve injury with a sub-SMAS and subplatysmal elevation should not be encountered. However, the risk of injury to the facial nerve and spinal accessory nerve may be increased when a deeper 
plane of dissection is utilized. Knowledge of cranial nerve anatomy is critical to avoid this preventable complication.

Previous techniques to allow sufficient laxity to cover large cheek defects have involved mobilizing large, anteriorly based rotation or advancement flaps. The incision lines descend the anterior border of the trapezius muscle crossing the clavicle onto the chest wall, becoming a cervicothoracic flap [14]. Flap elevation is noted to facilitate exposure when a parotidecotmy or neck dissection is required, as with many patients with malignant cheek lesions [18]. The flaps provide acceptable aesthetic results with scars hidden in the hairline and along natural contours. However, we believe that the cervicothoracic flap is not the optimal reconstructive technique for most moderate to large sized cheek defects. The infraclavicular elevation is time consuming to dissect, and we found it to be unnecessary in all of the cases in our series.

In this study, we describe a method for reconstructing moderate to large size cheek and external ear defects that is simple and reliable. The key points to our flap design are to achieve a tension-free closure of the defect by designing a flap that is the same width as the defect, to take the tip of the flap posterior to the hairline when necessary, and to make an inferior incision of the flap in a roughly horizontal line which is nearly always above the clavicle. Elevating the flap in a plane deep to the SMAS and platysma muscles is done to improve viability of the flap. With our technique, the greatest tension of the wound closure is at the less critical donor site rather than the defect. The series of patients that we have presented demonstrates that our method is a simple, quick, and reliable and results in good aesthetic outcomes for large cutaneous defects involving the cheek and external ear.

\section{REFERENCES}

[1] Esser JFS, Ed. Die Rotation der Wange. FVC Vogel Verlag 1918. Leipzig

[2] Kroll SS, Reece GP, Robb G, Black J. Deep-plane cervicofacial rotation-advancement flap for reconstruction of large cheek defects, Plast Reconstr Surg 1994; 1: 88-93.
[3] Cabrera R, Zide BM, Cheek Reconstruction: In: Aston SJ, Beasley RW, Thorne CHM, Eds. Grabb and Smith's Plastic Surgery, $5^{\text {th }}$ ed. Philadelphia: Lippincott 1999: pp. 501-512.

[4] Mitz V, Peyronie M. The Superficial Musculo-aponeurotic System (SMAS) in the parotid and cheek area. Plast Reconstr Surg. 1976; 1: 80-8.

[5] Zide BM. Deformities of the lips and cheeks. In: McCarthy JG, Ed. Plastic Surgery. Philadelphia: WB Saunders 1990: pp. 2009-2056.

[6] Menick FJ. Reconstruction of the cheek. Plast Reconstr Surg 2001; 2: 496-505.

[7] Stark RB, Kaplan JM. Rotation flaps, neck to cheek. Plast Reconstr Surg 1972; 3: 230-3.

[8] Rieck B, Giesler T. A medially based cervical transposition flap for soft tissue coverage of the cheek. Eur J Plast Surg 2004; 1: 20-23.

[9] Conley JJ. The use of regional flaps in head and neck surgery. Ann Otol Rhinol Laryngol 1960; 69: 1223-34.

[10] Kaplan I, Goldwyn RM. The versatility of the laterally based cervicofacial flap for cheek repairs. Plast Reconstr Surg 1978; 3: 390-3.

[11] Cook TA, Israel JM, Wang TD, Murakami CS, Brownrigg PJ. Cervical rotation flaps for midface resurfacing. Arch Otolaryngol Head Neck Surg 1991; 1: 77-82.

[12] Juri J, Juri C. Advancement and rotation of a large cervicofacial flap for cheek repairs. Plast Reconstr Surg 1979; 5: 692-6.

[13] Juri J, Juri C. Cheek reconstruction with advancement-rotation flaps. Clin Plast Surg 1981; 2: 223-6.

[14] Crow ML, Crow FJ. Resurfacing large cheek defects with rotation flaps from the neck. Plast Reconstr Surg 1976; 2: 196-200.

[15] Longaker MT, Glat PM, Zide BM. Deep plane cervicofacial "hike": anatomic basis with dog-ear blepharoplasty. Plast Reconstr Surg 1997; 1: 16-21.

[16] Skoog T. Plastic Surgery: New methods. Philadelphia: WB Saunders; 1974.

[17] Moore BA, Wine T, Netterville JL. Cervicofacial and cervicothoracic rotation flaps in head and neck reconstruction. Head Neck 2005; 12: 1092-101.

[18] Tan ST, MacKinnon CA. Deep plane cervicofacial flap: a useful and versatile technique in head and neck surgery. Head Neck 2006; 1: 46-55. 